Col I i si onl ess damping of geodesi c acoust i c modes

\begin{tabular}{|c|c|}
\hline $\begin{array}{l}\text { j our nal or } \\
\text { publ i cat i on } \mathrm{titl} \text { e }\end{array}$ & J our nal of Pl asma Physi cs \\
\hline vol une & Vol . 72 \\
\hline number & I ssue 6 \\
\hline page $r$ ange & pp. 825- 828 \\
\hline year & 2006-12-01 \\
\hline URL & ht t p: //hdl . handl e. net /10655/3830 \\
\hline
\end{tabular}




\title{
Collisionless damping of geodesic acoustic modes
}

\author{
H. S U G A M A and T.-H. W A T A N A B E \\ National Institute for Fusion Science/Graduate University for Advanced Studies, \\ Toki 509-5292, Japan
}

(Received 10 August 2005 and accepted 7 January 2006)

\begin{abstract}
Collisionless time evolutions of geodesic acoustic modes (GAMs) in tokamaks are investigated by the gyrokinetic theory and simulation. It is shown that the collisionless damping of the GAM oscillations is enhanced when the ratio of the typical drift orbit width of passing ions to the radial wavelength of the zonal flow increases.
\end{abstract}

\section{Introduction}

It was observed in collisionless gyrokinetic simulations that, in toroidal plasmas, the $\mathbf{E} \times \mathbf{B}$ zonal flow, which is added initially as an impulse, evolves as rapid oscillations of the geodesic acoustic mode (GAM) before it is damped and converges into a finite stationary value predicted theoretically [1-3]. The GAM was first predicted based on the fluid model [4]. Kinetic evaluations of frequencies and damping rates of the GAM were done in $[5,6]$ based on local drift kinetic models which do not include the magnetic drift term of the perturbed distribution function assuming the radial widths of ion drift orbits to be negligibly smaller than the radial wavelength of the fluctuation. In this paper, it is shown by the gyrokinetic analysis and simulation how the collisionless damping of the GAM oscillations in tokamaks is increased by the finite drift orbit widths of passing ions.

\section{Theoretical analysis}

The gyrokinetic equation for the zonal flow component with the perpendicular wave number vector $\mathbf{k}_{\perp}=k_{\mathrm{r}} \nabla r$ is given by [1]

$$
\left(\frac{\partial}{\partial t}+v_{\|} \mathbf{b} \cdot \nabla+i \omega_{\mathrm{D}}\right) \delta f_{\mathbf{k}_{\perp}}=-\left(v_{\|} \mathbf{b} \cdot \nabla+i \omega_{\mathrm{D}}\right)\left(F_{0} J_{0}\left(k_{\perp} \rho\right) \frac{e \phi_{\mathbf{k}_{\perp}}}{T}\right),
$$

where $\phi_{\mathbf{k}_{\perp}}$ is the electrostatic potential, $F_{0}$ is the Maxwellian equilibrium distribution function, $\rho$ is the gyroradius, $\Omega$ is the gyrofrequency, and $\omega_{\mathrm{D}} \equiv k_{\mathrm{r}} v_{\mathrm{dr}}$ is the drift frequency. Here, $w \equiv m v^{2} / 2$ and $\mu \equiv m v_{\perp}^{2} /(2 B)$ are used as independent velocity-space variables and subscripts to represent particle species are omitted. The perturbed gyrocenter distribution function $\delta f_{\mathbf{k}_{\perp}}$ consists of adiabatic and nonadiabatic parts, $\delta f_{\mathbf{k}_{\perp}}=-F_{0} J_{0}\left(k_{\perp} \rho\right)\left(e \phi_{\mathbf{k}_{\perp}} / T\right)+g_{\mathbf{k}_{\perp}}$. For large-aspect-ratio tokamaks, the magnetic field strength is given by $B=B_{0}(1-\epsilon \cos \theta)$ where $\theta$ is the poloidal angle, $\epsilon \equiv r / R_{0}$ is the inverse aspect ratio and $r\left(R_{0}\right)$ is the minor (major) radius. Hereafter, $v_{\|}$is used as the independent variable instead of $w$. We now consider passing ions, for which we neglect the mirror force term and rewrite (2.1) as

$$
\left(\frac{\partial}{\partial t}+\frac{v_{\|}}{R_{0} q} \frac{\partial}{\partial \theta}\right)\left(e^{i k_{\mathrm{r}} \hat{\delta} \cos \theta} \delta \hat{f}_{\mathbf{k}_{\perp}}\right)=-\frac{v_{\|}}{R_{0} q} \frac{\partial}{\partial \theta}\left(e^{i k_{\mathrm{r}} \hat{\delta} \cos \theta} J_{0} \frac{e \phi_{\mathbf{k}_{\perp}}}{T}\right),
$$


where $\delta \hat{f}_{\mathbf{k}_{\perp}} \equiv \delta f_{\mathbf{k}_{\perp}} / F_{0}, q \equiv r B_{0} /\left(R_{0} B_{\mathrm{P}}\right), \hat{\delta} \equiv\left(\epsilon / \Omega_{\mathrm{P}}\right)\left[v_{\|}+\mu B_{0} /\left(m v_{\|}\right)\right]$, and $\Omega_{\mathrm{P}}=$ $e B_{\mathrm{P}} /(m c)$. Here, $\hat{\delta} \cos \theta$ represents the radial dispacement of the passing ion. Using Fourier and Laplace transforms with respect to $\theta$ and $t$, respectively, as $\left[\delta \hat{f}_{\mathbf{k}_{\perp}}(\theta, t)\right.$, $\left.\phi_{\mathbf{k}_{\perp}}(\theta, t)\right]=\sum_{m}(2 \pi)^{-1} \int d \omega e^{i m \theta-i \omega t}\left[\delta \hat{f}_{k_{\mathrm{r}}, m}(\omega), \phi_{k_{\mathrm{r}}, m}(\omega)\right]$, we obtain from (2.2),

$$
\begin{aligned}
\delta \hat{f}_{k_{\mathrm{r}}, m}(\omega)= & \sum_{l, l^{\prime}} i^{l^{\prime}-l} J_{l}\left(k_{\mathrm{r}} \hat{\delta}\right) J_{l^{\prime}}\left(k_{\mathrm{r}} \hat{\delta}\right)\left(\frac{(m+l)\left(v_{\|} / R_{0} q\right)}{\omega-(m+l)\left(v_{\|} / R_{0} q\right)}\right)\left(\frac{e \phi_{k_{\mathrm{r}}, m+l-l^{\prime}}(\omega)}{T}\right) \\
& +\delta \hat{I}_{k_{\mathrm{r}}, m}(\omega),
\end{aligned}
$$

for $m \neq 0$ Fourier components, where $\delta \hat{I}_{k_{\mathrm{r}}, m}(\omega)$ denotes the initial condition term. We see that, for finite orbit widths $\left|k_{\mathrm{r}} \hat{\delta}\right|>0, l \neq 0$ terms generate the resonance conditions $\omega-(m+l)\left(v_{\|} / R_{0} q\right)=0$ which can influence the GAM damping rate. However, the zero-gyroradius limit $k_{\perp} \rho \rightarrow 0$ is taken in deriving (2.3). The quasineutrality condition is given by $\int d^{3} v F_{0} \delta \hat{f}_{\mathrm{i} k_{\mathrm{r}} m}-n_{0}\left(k_{\mathrm{r}} a_{\mathrm{i}}\right)^{2} e \phi_{k_{\mathrm{r}} m} / T_{\mathrm{i}}=\delta n_{\mathrm{e} k_{\mathrm{r}} m}$ with $a_{\mathrm{i}} \equiv\left(T_{\mathrm{i}} / m_{\mathrm{i}}\right)^{1 / 2} / \Omega_{\mathrm{i}}$. The perturbed electron density is given by $\delta n_{\mathrm{e} k_{\mathrm{r}} m}=$ $n_{0} e \phi_{k_{\mathrm{r}} m} / T_{\mathrm{e}}$ (for $m \neq 0$ ), 0 (for $m=0$ ). Here, subscripts referring to particle species are explicitly shown. For $k_{\mathrm{r}} a_{\mathrm{i}} \ll 1$, we obtain

$$
\int d^{3} v F_{0} \delta \hat{f}_{\mathrm{i} k_{\mathrm{r}} m}(\omega)=n_{0} \frac{e \phi_{k_{\mathrm{r}} m}(\omega)}{T_{\mathrm{e}}} \quad(m \neq 0) .
$$

Combining the quasineutrality condition with (2.1) gives

$$
\begin{aligned}
& n_{0}\left(k_{\mathrm{r}} a_{\mathrm{i}}\right)^{2} \frac{e}{T_{\mathrm{i}}}\left[-i \omega \phi_{k_{\mathrm{r}} 0}(\omega)-\phi_{k_{\mathrm{r}} 0}(t=0)\right] \\
& \quad=\int d^{3} v F_{0} \frac{k_{\mathrm{r}}}{2 R_{0} \Omega_{\mathrm{i}}}\left(v_{\|}^{2}+\frac{\mu B_{0}}{m_{\mathrm{i}}}\right)\left[\delta \hat{f}_{\mathrm{i} k_{\mathrm{r}}-1}(\omega)+\frac{e \phi_{k_{\mathrm{r}}-1}(\omega)}{T_{\mathrm{i}}}-\delta \hat{f}_{\mathrm{i} k_{\mathrm{r}} 1}(\omega)-\frac{e \phi_{k_{\mathrm{r}} 1}(\omega)}{T_{\mathrm{i}}}\right] .
\end{aligned}
$$

Using the symmetry property of $(2.3)-(2.5)$, we can seek the solution for the GAM which satisfies $\delta \hat{f}_{\mathrm{i} k_{\mathrm{r}} m}\left(v_{\|}\right)=(-1)^{m} \delta \hat{f}_{\mathrm{i} k_{\mathrm{r}}-m}\left(-v_{\|}\right)$and $\phi_{k_{\mathrm{r}} m}=(-1)^{m} \phi_{k_{\mathrm{r}}-m}$. Then we find from $(2.3)$ and $(2.4)$ that $\phi_{k_{\mathrm{r}} m} / \phi_{k_{\mathrm{r}} 0} \sim\left(k_{\mathrm{r}} \hat{\delta}\right)^{m}(m=1,2, \ldots)$ for $\left|k_{\mathrm{r}} \hat{\delta}\right| \ll 1$. Also, from (2.3) with $m=1$, we have

$$
\begin{aligned}
\delta \hat{f}_{\mathrm{i} k_{\mathrm{r}} 1}(\omega)= & {\left[\frac{\left(v_{\|} / R_{0} q\right)}{\omega-\left(v_{\|} / R_{0} q\right)}+\left(\frac{k_{\mathrm{r}} \hat{\delta}}{2}\right)^{2} \frac{2\left(v_{\|} / R_{0} q\right)}{\omega-2\left(v_{\|} / R_{0} q\right)}\right] } \\
& \times\left[\frac{e \phi_{k_{\mathrm{r}} 1}(\omega)}{T_{\mathrm{i}}}+i\left(\frac{k_{r} \hat{\delta}}{2}\right) \frac{e \phi_{k_{\mathrm{r}} 0(\omega)}}{T_{\mathrm{i}}}\right]+\delta \hat{I}_{\mathrm{i} k_{\mathrm{r}} 1}(\omega),
\end{aligned}
$$

where terms including $\phi_{k_{\mathrm{r}} m}$ with $m \geqslant 2$ are negleted. Here, we see two resonance conditions $\omega=v_{\|} / R_{0} q$ and $\omega=2 v_{\|} / R_{0} q$, in which the latter may seem to be ineffective due to small factors of a higher order in $k_{\mathrm{r}} \hat{\delta}$ appearing together with the factor $1 /\left[\omega-2\left(v_{\|} / R_{0} q\right)\right]$ although its influence can dominate the GAM damping because the population of ions with the resonant parallel velocity $v_{\|}=R_{0} q \omega / 2$ becomes larger than that with $v_{\|}=R_{0} q \omega$. Thus, in (2.6), we retain the resonant (or imaginary) part of $1 /\left[\omega-2\left(v_{\|} / R_{0} q\right)\right]$ while neglecting its non-resonant (or real) part. Then, when using (2.6) for evaluations of velocity-space integrals, we perform the replacement, $1 /\left[\omega-2\left(v_{\|} / R_{0} q\right)\right] \rightarrow-i \pi \delta\left[\omega_{\mathrm{r}}-2\left(v_{\|} / R_{0} q\right)\right]$, where $\left|\omega_{\mathrm{i}} / \omega_{\mathrm{r}}\right| \ll 1$ with $\left(\omega_{\mathrm{r}}, \omega_{\mathrm{i}}\right) \equiv(\operatorname{Re}(\omega), \operatorname{Im}(\omega))$ is assumed. We note from the symmetry condition 
that other resonances $\omega=-v_{\|} / R_{0} q$ and $\omega=-2 v_{\|} / R_{0} q$ appear in the equation for $\delta \hat{f}_{k_{\mathrm{r}}-1}(\omega)$.

We now assume the initial perturbed ion gyrocenter distribution function to take the Maxwellian form, $\delta f_{i \mathbf{k}_{\perp}}(t=0)=\left(\delta n_{\mathbf{i k}_{\perp}}^{\text {(gyro) }}(t=0) / n_{0}\right) F_{i 0}$, where $\delta n_{\mathbf{i k}_{\perp}}^{\text {(gyro) }}(t=$ $0)=n_{0}\left(k_{\perp}^{2} a_{\mathrm{i}}^{2}\right) e \phi_{k_{\mathrm{r}} 0}(t=0) / T_{\mathrm{i}}$. In this case, the effect of $\delta \hat{I}_{\mathrm{i} k_{\mathrm{r}} 1}(\omega)$ in $(2.6)$ on the the zonal-flow potential evolution is negligibly small compared with that of $\phi_{k_{\mathrm{r}} 0}(t=0)$ in (2.5). Then, using (2.4)-(2.6), we obtain $\phi_{k_{\mathrm{r}} 0}(\omega)=K(\omega) \phi_{k_{\mathrm{r}} 0}(t=0)$, where

$$
\begin{aligned}
& \frac{1}{K(\omega)}=-i \hat{\omega}-i \frac{q^{2}}{2}\left[2 \hat{\omega}^{3}+3 \hat{\omega}+\left(2 \hat{\omega}^{4}+2 \hat{\omega}^{2}+1\right) Z(\hat{\omega})-\frac{\hat{\omega}}{2}\left\{2 \hat{\omega}+\left(2 \hat{\omega}^{2}+1\right) Z(\hat{\omega})\right\}^{2}\right. \\
& \quad \times\left\{\frac{T_{\mathrm{i}}}{T_{\mathrm{e}}}+1+\hat{\omega} Z(\hat{\omega})\right\}^{-1}+i \frac{\sqrt{\pi}}{2}\left(\frac{k_{\mathrm{r}} v_{T_{\mathrm{i}}} q}{\Omega_{\mathrm{i}}}\right)^{2} e^{-\hat{\omega}_{\mathrm{r}}^{2} / 4}\left\{\frac{\hat{\omega}_{\mathrm{r}}^{6}}{64}+\left(\frac{\hat{\omega}_{\mathrm{r}}^{4}}{8}+\frac{3 \hat{\omega}_{\mathrm{r}}^{2}}{4}+3\right.\right. \\
& \left.\left.\left.\quad+\frac{6}{\hat{\omega}_{\mathrm{r}}^{2}}\right)\left(1-\frac{3 \hat{\omega}_{\mathrm{r}}}{16}\left\{2 \hat{\omega}_{\mathrm{r}}+\left(2 \hat{\omega}_{\mathrm{r}}^{2}+1\right) Z_{\mathrm{r}}\left(\hat{\omega}_{\mathrm{r}}\right)\right\}\left\{\frac{T_{\mathrm{i}}}{T_{\mathrm{e}}}+1+\hat{\omega}_{\mathrm{r}} Z_{\mathrm{r}}\left(\hat{\omega}_{\mathrm{r}}\right)\right\}^{-1}\right)\right\}\right] .
\end{aligned}
$$

Here, $Z(\hat{\omega})$ is the plasma dispersion function of the normalized frequency $\hat{\omega} \equiv$ $R_{0} q \omega / v_{T_{\mathrm{i}}}\left(v_{T_{\mathrm{i}}} \equiv \sqrt{2 T_{\mathrm{i}} / m_{\mathrm{i}}}\right)$ and the subscript $r$ represents the real part. The last group of terms proportional to $\left(k_{\mathrm{r}} v_{T_{\mathrm{i}}} q / \Omega_{\mathrm{i}}\right)^{2} \exp \left(-\hat{\omega}_{\mathrm{r}}^{2} / 4\right)$ of the right-hand side of (2.7) appear due to the resonance of passing ions with the GAM at $\left|v_{\|}\right|=R q \omega / 2(=$ $\left.v_{T_{\mathrm{i}}} \hat{\omega} / 2\right)$ caused by the finite orbit widths. Contributions from other resonances at $\left|v_{\|}\right|=R q \omega / n(n=3,4,5, \ldots)$ are proportional to $\left(k_{\mathrm{r}} v_{T_{\mathrm{i}}} q / \Omega_{\mathrm{i}}\right)^{n} \exp \left(-\hat{\omega}_{\mathrm{r}}^{2} / n^{2}\right)$ and neglected in (2.7). The inverse Laplace transform of $\phi_{k_{\mathrm{r}} 0}(\omega)=K(\omega) \phi_{k_{\mathrm{r}} 0}(t=0)$ gives $\phi_{k_{\mathrm{r}} 0}(t)=K(t) \phi_{k_{\mathrm{r}} 0}(0)$ where $K(t)=(2 \pi i)^{-1} \int d \omega e^{-i \omega t} K(\omega)$. Using the pair of poles $\omega= \pm \omega_{\mathrm{G}}+i \gamma$ for $K(\omega)$ which correspond to the minimum damping rate, $\phi_{k_{\mathrm{r}} 0}(t)=K(t) \phi_{k_{\mathrm{r}} 0}(0)$ is approximately written as $\phi(t)=\phi(0) \cos \left(\omega_{\mathrm{G}} t\right) \exp (\gamma t)$. Recall that $K(t)$ describes the short-time behavior of the zonal-flow potential, in which slower variations than the GAM oscillations are neglected. These slow behaviors are described by the Rosenbluth-Hinton theory [1], in which an essential role is played by trapped particles that are ignored in (2.3). Taking account of the residual zonal flow in the collisionless long-time behavior, we can write

$$
\phi_{k_{\mathrm{r}} 0}(t)=\phi_{k_{\mathrm{r}} 0}(\infty)+\left[\phi_{k_{\mathrm{r}} 0}(0)-\phi_{k_{\mathrm{r}} 0}(\infty)\right] \cos \left(\omega_{\mathrm{G}} t\right) \exp (\gamma t),
$$

where $\phi_{k_{\mathrm{r}} 0}(\infty)=\phi_{k_{\mathrm{r}} 0}(0) /\left(1+1.6 q^{2} / \epsilon^{1 / 2}\right)$ is the undamped component of the initial zonal flow derived by Rosenbluth and Hinton [1]. When $\hat{\omega}_{\mathrm{G}}^{2} \equiv\left(R_{0} q \omega_{\mathrm{G}} / v_{T_{\mathrm{T}}}\right)^{2} \gg 1$ and $\tau_{\mathrm{e}} \equiv T_{\mathrm{e}} / T_{\mathrm{i}}$ are used, approximate expressions for $\omega_{\mathrm{G}}$ and $\gamma$ are obtained as

$$
\begin{aligned}
\omega_{\mathrm{G}}= & \frac{\sqrt{7+4 \tau_{\mathrm{e}}}}{2} q\left(\frac{v_{T_{\mathrm{i}}}}{R_{0} q}\right)\left[1+\frac{2\left(23+16 \tau_{\mathrm{e}}+4 \tau_{\mathrm{e}}^{2}\right)}{q^{2}\left(7+4 \tau_{\mathrm{e}}\right)^{2}}\right]^{1 / 2} \\
\gamma= & -\frac{\sqrt{\pi}}{2} q^{2}\left(\frac{v_{T_{\mathrm{i}}}}{R_{0} q}\right)\left[1+\frac{2\left(23 / 4+4 \tau_{\mathrm{e}}+\tau_{\mathrm{e}}^{2}\right)}{q^{2}\left(7 / 2+2 \tau_{\mathrm{e}}\right)^{2}}\right]^{-1}\left[\exp \left(-\hat{\omega}_{\mathrm{G}}^{2}\right)\left\{\hat{\omega}_{\mathrm{G}}^{4}+\left(1+2 \tau_{\mathrm{e}}\right) \hat{\omega}_{\mathrm{G}}^{2}\right\}\right. \\
& \left.+\frac{1}{4}\left(\frac{k_{\mathrm{r}} v_{T_{\mathrm{i}}} q}{\Omega_{\mathrm{i}}}\right)^{2} \exp \left(-\hat{\omega}_{\mathrm{G}}^{2} / 4\right)\left\{\frac{\hat{\omega}_{\mathrm{G}}^{6}}{64}+\left(1+\frac{3}{8} \tau_{\mathrm{e}}\right)\left(\frac{\hat{\omega}_{\mathrm{G}}^{4}}{8}+\frac{3 \hat{\omega}_{\mathrm{G}}^{2}}{4}\right)\right\}\right] .
\end{aligned}
$$

\section{Numerical results and discussion}

A time evolution of the zonal-flow potential obtained by the gyrokinetic-Vlasov simulation [3] for $\epsilon=0.1, \tau_{\mathrm{e}}=1, q=1.5$ and $k_{\mathrm{r}} a_{\mathrm{i}}=0.131$ is plotted by open circles 


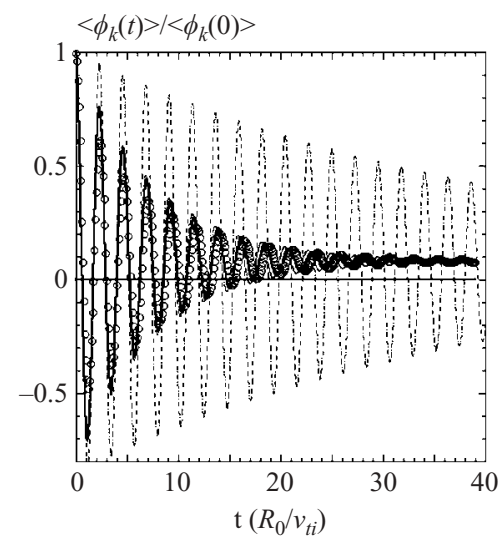

Figure 1. Zonal-flow potential as a function of time. Here $v_{t_{\mathrm{i}}} \equiv\left(T_{\mathrm{i}} / m_{\mathrm{i}}\right)^{1 / 2}$.

in Fig. 1. The analytical results from (2.8) are also plotted in Fig. 1, where a thin solid curve is obtained by using (2.7) to numerically search solutions $\omega=\omega_{\mathrm{G}}+i \gamma$ of $1 / K(\omega)=0$ while the approximate expressions for $\left(\omega_{\mathrm{G}}, \gamma\right)$ in $(2.9)$ and $(2.10)$ are used to plot a thick solid curve. A good agreement between the analytical predictions and the simulation results on the GAM frequencies and damping rates is verified. A dotted curve in Fig. 1, which shows a significantly slower damping, is obtained by neglecting the finite-orbit-width effect terms proportional to $\left(k_{\mathrm{r}} v_{T_{\mathrm{i}}} q / \Omega_{\mathrm{i}}\right)^{2}$ in $(2.7)$. The reason of the damping-rate enhancement by the finite orbit width is explained as follows. When the ratio of the typical orbit width of passing ions to the radial wavelength of the zonal flow increases, the radial magnetic drift of the perturbed ion gyrocenter distribution strengthens the poloidal mode number coupling and grows the oscillation component with the poloidal wave number doubled. Since the parallel velocity required to resonate with the GAM is lowered, this higher poloidal wave number component yields a significant population of resonant ions and increases the resonance damping of the GAM.

\section{Acknowledgements}

This work is supported in part by the Japanese Ministry of Education, Culture, Sports, Science, and Technology, Grant Nos. 16560727 and 17360445 and in part by the NIFS Collaborative Research Program, NIFS04KDAD003 and NIFS04KNXN023.

\section{References}

[1] Rosenbluth, M. N. and Hinton, F. L. 1998 Phys. Rev. Lett. 80, 724.

[2] Sugama, H. and Watanabe, T.-H. 2005 Phys. Rev. Lett. 94, 115001.

[3] Watanabe, T.-H. and Sugama, H. 2004 20th IAEA Fusion Energy Conference, In Proceedings of the Vilamoura, Portugal, TH/8-3Rb.

[4] Winsor, N., Johnson, J. L. and Dawson, J. J. 1968 Phys. Fluids 11, 2248.

[5] Lebedev, V. B. et al. 1996 Phys. Plasmas 3, 3023.

[6] S. V. Novakovskii, et al. 1997 Phys. Plasmas 4, 4272. 